\title{
Optical properties of nano-silicon
}

\author{
S TRIPATHY, R K SONI*, S K GHOSHAL and K P JAIN \\ Department of Physics, Indian Institute of Technology, New Delhi 110 016, India
}

\begin{abstract}
We investigated the optical properties of silicon clusters and Si nanocrystallites using photoluminescence (PL) and Raman scattering technique. Broad luminescence band in the red region was observed from $\mathrm{Si}$-doped $\mathrm{SiO}_{2}$ thin films deposited by co-sputtering of $\mathrm{Si}$ and $\mathrm{SiO}_{2}$ on $p$-type $\mathrm{Si}(100)$ substrates, annealed in $\mathrm{Ar}$ and $\mathrm{O}_{2}$ atmosphere. Nanocrystalline $\mathrm{Si}$ particles fabricated by pulsed plasma processing technique showed infrared luminescence from as grown film at room temperature. Raman spectra from these films consisted of broad band superimposed on a sharp line near $516 \mathrm{~cm}^{-1}$ whose intensity, frequency, and width depend on the particle sizes arising from the phonon confinement in the nanocrystalline silicon. We also performed PL, Raman and resonantly excited PL measurements on porous silicon film to compare the optical properties of $\mathrm{Si}$ nanostructures grown by different techniques. An extensive computer simulation using empirical pseudopotential method was carried out for 5-18 atoms Si clusters and the calculated gap energies were close to our PL data.
\end{abstract}

Keywords. Nanocrystalline silicon; Raman scattering; photoluminescence; porous silicon.

\section{Introduction}

Desire for the integration of silicon nanostructures in optical devices has led to the search for silicon-based materials and structures that emit light with high quantum efficiency. Recently, the discovery of luminescence from Si nanostructures by Canham (1990) has attracted much attention towards the quantum mechanical nature of this phenomenon. The optical and electronic properties of the materials are not yet fully understood. Surface effects (large surface to volume ratio) as well as quantum confinement effects are considerably enhanced in silicon nanostructures which control the photoluminescence. Silicon quantum dots have attracted intense theoretical and experimental investigations in recent years. The importance of such an investigation stems from the fact that the modeling of such novel materials requires a fundamental understanding of the electronic structure including the role played by surface having different geometry, disorder, inhomogeneity and so on. Thus, inspite of intensive studies by Kanemitsu (1995) and John and Singh (1995), no conclusive argument has been given on the mechanisms of efficient light emission from porous silicon and related materials. In porous silicon the luminescence properties may be related to different silicon compounds such as $a$-Si: $\mathrm{H}$, polysilane, $\mathrm{SiH}_{x}$ and surface defect states. One of the explanations given by Takagahara and Takeda (1996), for the blue-shifted photoluminescence

\footnotetext{
*Author for correspondence

†Paper presented at the 5th IUMRS ICA98, October 1998, Bangalore
}

from porous silicon is that the microstructure consists of islands of isolated quantum dots, each experiencing quantum confinement. Experimental efforts to create silicon-based light-emitting films have been made applying various techniques such as sputtering, plasma processing and anodic etching. Morisaki et al (1991) reported the visible light luminescence from some other form of $\mathrm{Si}$ nanostructures such as Si ultrafine particles deposited by evaporation of silicon powders in an $\mathrm{Ar}$ atmosphere. Visible luminescence from $\mathrm{Si}$ ultrafine particles embedded in $\mathrm{SiO}_{2}$ was also reported by Osaka et al (1992). Since the silicon surface in the $\mathrm{Si}$-doped $\mathrm{SiO}_{2}$ is protected from contamination and natural oxidation in air, it is expected to show more stable luminescence than other Si nanostructures.

In this report we studied luminescence mechanism in the $\mathrm{Si}$-doped $\mathrm{SiO}_{2}$ thin films prepared by $\mathrm{RF}$ sputtering of a target consisting of $\mathrm{Si}$ pieces placed on a $\mathrm{SiO}_{2}$ substrate in $\mathrm{Ar}$ and $\mathrm{O}_{2}$ atmosphere. Raman scattering and PL studies were also performed on nanocrystalline silicon prepared by pulsed plasma processing. For comparative study, Raman and PL measurements were performed on porous silicon prepared by simple anodic etching technique. In order to explain the luminescence results, we have used the empirical pseudopotential method to calculate the electronic structure of Si quantum dots of different sizes. We found an enhancement of the calculated gap energy when the surface of the dot was passivated with the hydrogen atoms incorporating proper hydrogen pseudopotential.

\section{Experimental}

PL measurements were performed on the $\mathrm{Si}$-doped $\mathrm{SiO}_{2}$ thin films deposited on $p$-type $\mathrm{Si}$ (100) substrates by 
co-sputtering of $\mathrm{Si}$ and $\mathrm{SiO}_{2}$. The sputtering targets were 16 and $36 \mathrm{Si}$ pieces $(5 \mathrm{~mm} \times 5 \mathrm{~mm})$ placed on $\mathrm{SiO}_{2}$ substrates to vary the compositional fraction of $\mathrm{Si} / \mathrm{SiO}_{2}$ in the samples. The samples were annealed in $\mathrm{Ar}$ and $\mathrm{O}_{2}$ atmosphere at temperatures ranging from 300 to $1100^{\circ} \mathrm{C}$. PL and Raman measurements were also performed on spherical shaped nanocrystalline $\mathrm{Si}$ clusters less than $10 \mathrm{~nm}$, grown by pulsed gas supply of $\mathrm{SiH}_{4}$ and $\mathrm{H}_{2}$ in very high frequency (VHF) plasma. Porous silicon was grown in the dark on a (100) oriented $p$-Si wafer with resistivity $8 \Omega \mathrm{cm}$, by anodic etching in a HF solution for $30 \mathrm{~min}$ etch time at a current density of $10 \mathrm{~mA} / \mathrm{cm}^{2}$. PL and Raman scattering were excited in these nanometer sized silicon particles using $\mathrm{Ar}^{+}$laser and the signal was dispersed using a double monochromator. Conventional photon counting electronics was used to record the spectra. Resonantly excited PL spectra were taken using $647 \mathrm{~nm}$ line of $\mathrm{Kr}^{+}$laser where the incident laser power was kept below $20 \mathrm{~mW}$.

\section{Results and discussion}

Figure 1 shows PL spectra of $\mathrm{Si}$-doped $\mathrm{SiO}_{2}$ thin films annealed in $\mathrm{O}_{2}$ and $\mathrm{Ar}$ atmosphere with 16 and 36 piece silicon targets. Broad luminescence band in the red region

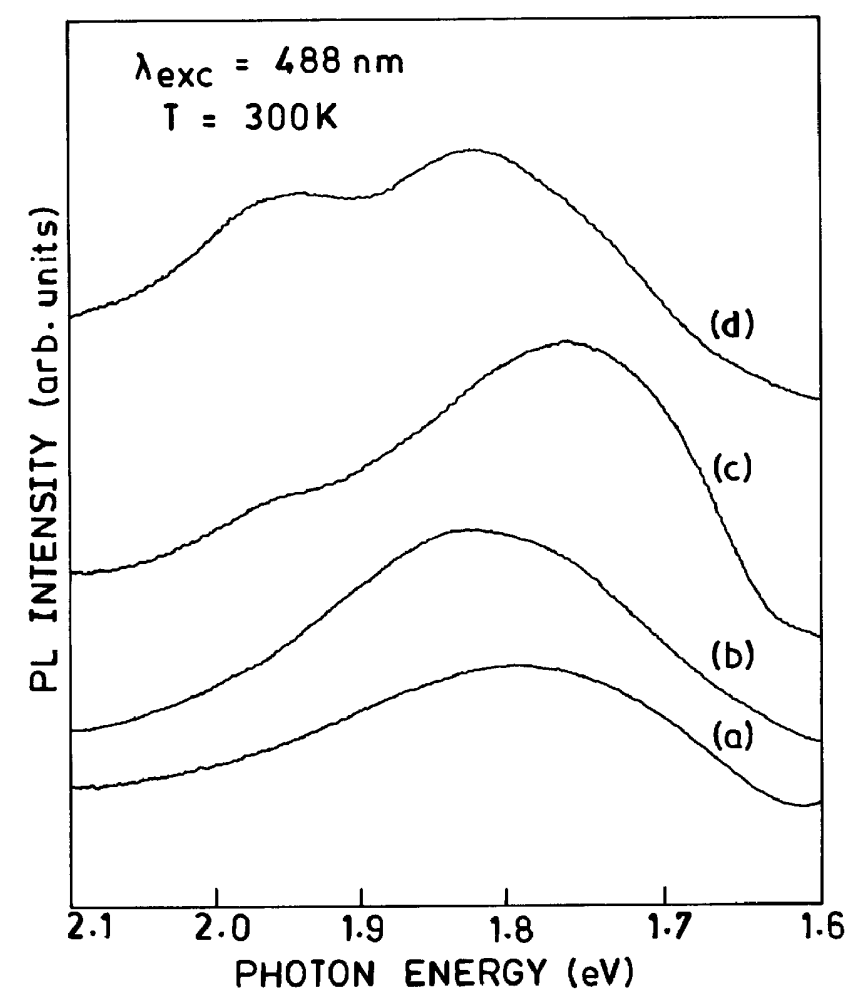

Figure 1. $\mathrm{PL}$ spectra of $\mathrm{Si}$-doped $\mathrm{SiO}_{2}$ thin films under different preparation conditions. (a) 36 pieces of $\mathrm{Si}$ targets annealed in $\mathrm{Ar}$, (b) 36 pieces of Si targets annealed in $\mathrm{O}_{2}$, (c) 16 pieces of Si target annealed in Ar and (d) 16 pieces of Si targets annealed in $\mathrm{O}_{2}$. was observed in the annealed samples. The emission band resembles that of porous silicon. The peak positions vary with annealing condition for the same $\mathrm{Si} / \mathrm{SiO}_{2}$ compositions. It shows PL emission process occurring at higher wavelengths for sample annealed under Ar, than those annealed under $\mathrm{O}_{2}$ atmosphere, when annealing temperature was increased. We believe that the luminescence does not reflect the band-to-band transitions and involves radiative recombination via recombination centres. As the radiative recombination centres may be associated with the surface states in the nanocrystalline silicon, the PL peak energy will be smaller than the band gap of a $\mathrm{Si}$ nanocrystal. The large broadening in the PL spectra can be associated with a large distribution of particle size in the grown film. The PL of the dot ensemble is due to the fluctuation in dot size, shape and strain corresponding to the statistical distribution of the eigen energies. Considering PL gap energy varying as $1 / d^{2}$, where ' $d$ ' is size of the nanocrystallite, the particle sizes are estimated to be $3.5 \mathrm{~nm}$ to $4.5 \mathrm{~nm}$ in these samples using first principle calculation. The particle sizes are smaller for the samples annealed in $\mathrm{O}_{2}$ prepared by 16 piece silicon targets.

Figure 2 shows PL spectra from $\mathrm{Si}$ nanocrystallites grown by pulsed plasma technique on quartz substrates. The infrared PL is observed from the as grown film at room temperature. The fine silicon particles $(4-10 \mathrm{~nm}$ size) grown under this condition shows good control on particle size distribution. Infrared absorption measurements have clarified that the surface of these nanocrystalline silicon particles is covered by hydrogen. Experimentally it has been found that the crystalline size increases with increasing the hydrogen plasma treatment

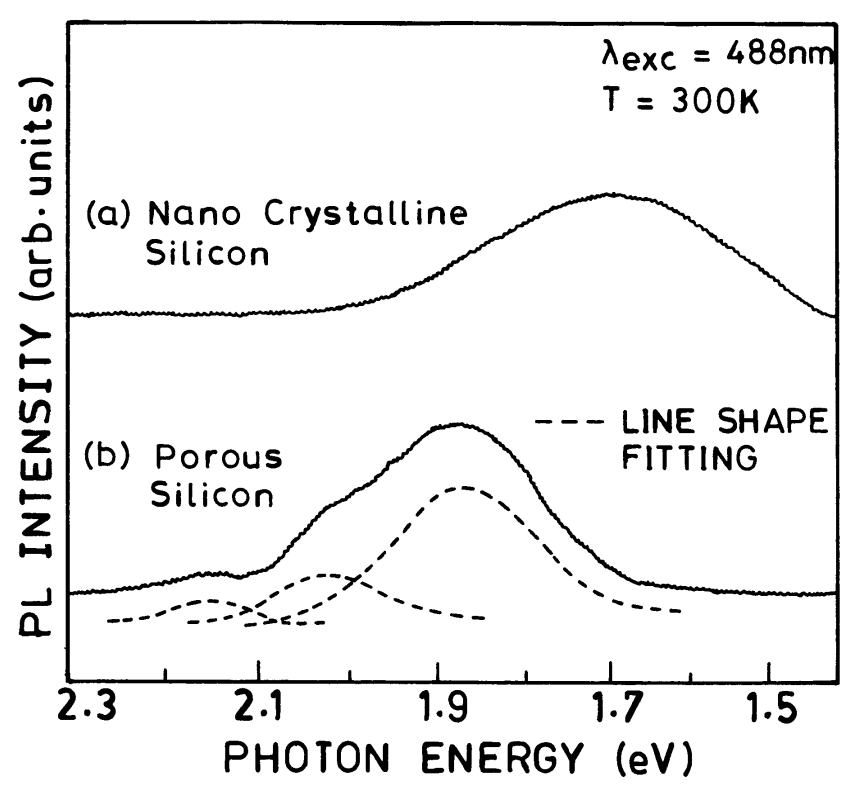

Figure 2. Room temperature Raman spectra of (a) nanocrystalline $\mathrm{Si}$ prepared by pulsed plasma processing and (b) porous silicon prepared by anodic etching using $488 \mathrm{~nm}$ line of $\mathrm{Ar}^{+}$laser. 
time or with decreasing the thickness of the $a$-Si : H layer. This results in termination of dangling bonds of the surface $\mathrm{Si}$ atoms of nanocrystalline silicon. Nanocrystalline silicon thus fabricated is embedded in an $a$ - $\mathrm{Si}: \mathrm{H}$ matrix. We have studied the phonon states in these nanocrystalline silicon using Raman scattering. The Raman spectra shown in figure 3 from this sample consists of a broad band superimposed on a sharp line near $516 \mathrm{~cm}^{-1}$. We argue that the sharp line, whose intensity, frequency, and width depend on the particle size, arises from the phonon confinement in nanometer sized crystalline silicon. The broad Raman band resembles that of density-of-state spectrum in amorphous silicon and indicates the presence of amorphous silicon like structure in the film. The disorder in the surface layer of the silicon particles is attributed to the amorphous structure. For comparative study, the PL and Raman spectra from porous silicon film is also

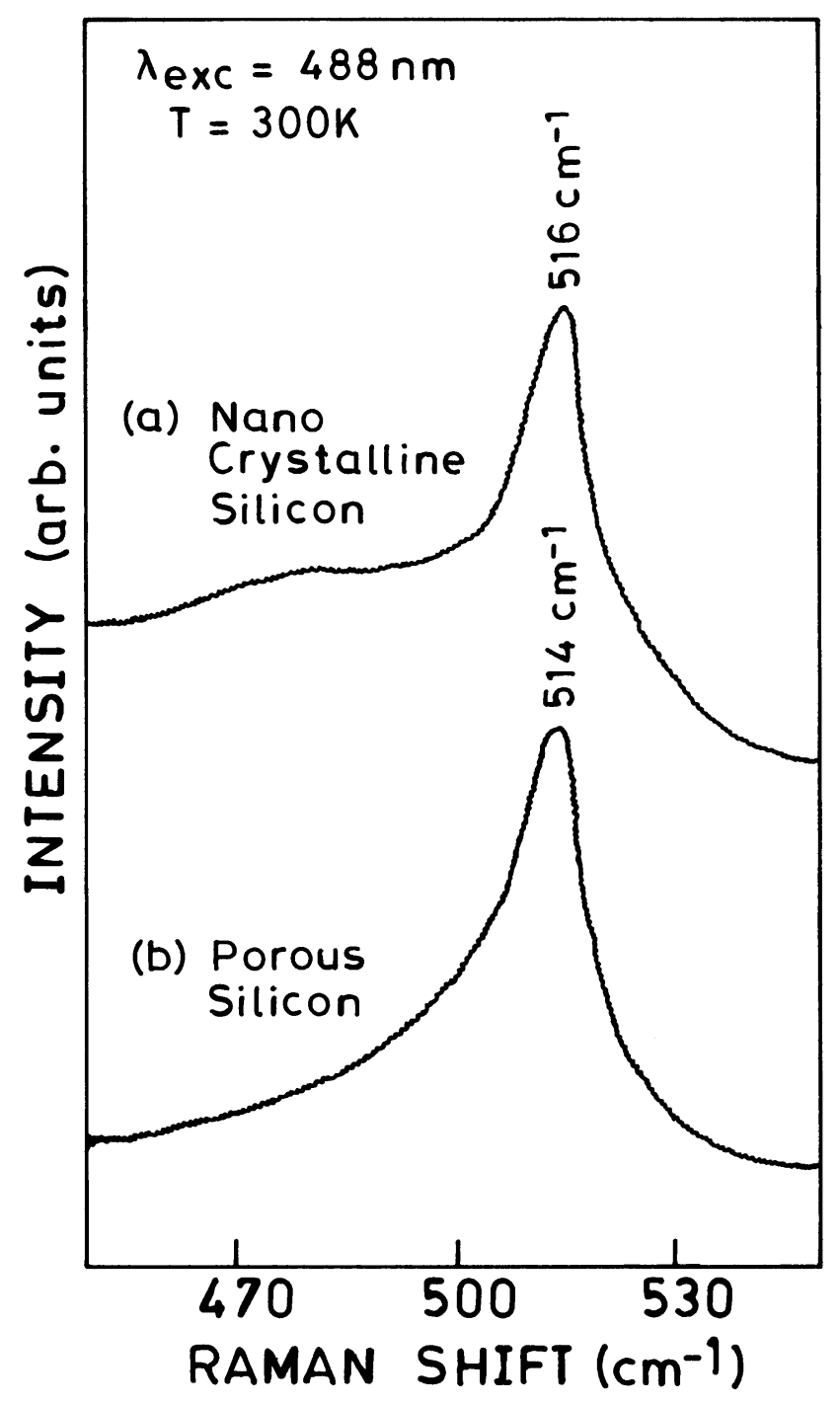

Figure 3. PL spectra of (a) nanocrystalline silicon and (b) porous silicon. The multiple peak PL spectrum in porous silicon is fitted with combination of three Gaussian distribution functions. shown in figures 2 and 3 . The size dependence of the Raman spectra is analyzed using spatial correlation model which takes into account the discrete wave vector of the confined phonon. The peak position of the strong Raman line at $514 \mathrm{~cm}^{-1}$ observed in the case of porous silicon layer undergoes very small changes as compared to those of crystalline silicon. The asymmetrically broadened line shape was studied using a model of phonon confinement proposed by Campbell and Fauchet (1986) which suggests that local structure of porous silicon is more likely a sphere than a rod and has a characteristic diameter $3 \cdot 5-5 \cdot 5 \mathrm{~nm}$.

The photo-excited light emission in porous silicon is characterized by a broad band in the visible region as evident from PL spectra excited with $488 \mathrm{~nm}(2.54 \mathrm{eV})$ laser line. The higher energy excitation leads to convolution of large quantized bands (more discrete states) in the conduction band giving rise to multiple broad peaks in the PL spectrum, which is also attributed to highly disordered local structure. It has been proposed that shift of the luminescence from infrared to visible region of porous silicon is caused by the band gap widening resulting from the quantum size effects. Whether the porous silicon skeleton basically consists of $\mathrm{Si}$ wires or nanoparticles or even a combination of both, we believe that the steady state PL at room temperature is also controlled by the radiative recombination of carriers in the surface-localized states. The radiative recombination of the localized electrons trapped by the surface states located within the band gap of porous silicon with its partner in the valence band and the radiative recombination of photon-excited electronhole pairs trapped in the crystalline nanostructures as

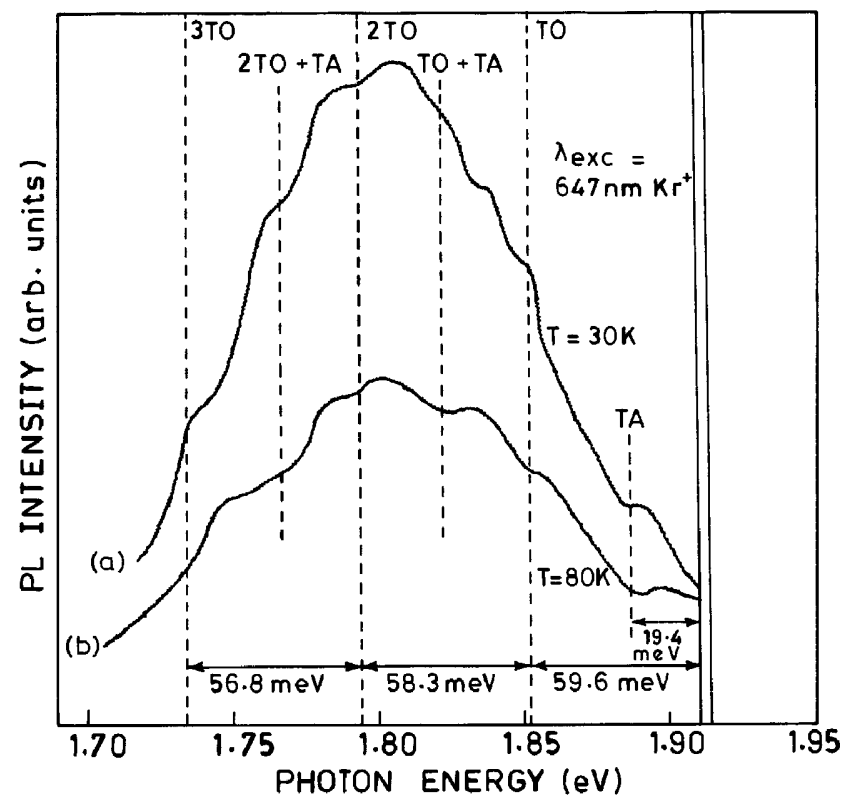

Figure 4. Resonantly excited PL spectra from porous silicon using $647 \mathrm{~nm}$ line of $\mathrm{Kr}^{+}$laser at (a) $30 \mathrm{~K}$ and (b) $80 \mathrm{~K}$. Step onset phonon energy separations are estimated from the model proposed by Suemoto et al (1993). 
excitons, resulted into multiple luminescence peaks. To further explain the PL mechanism resonant excitation was performed using $1.916 \mathrm{eV}$ line of $\mathrm{Kr}^{+}$laser. Figure 4 shows resonantly excited PL spectra with step like phonon structures (energy difference $56 \pm 2 \mathrm{meV}$ between each step) at two different temperatures. At different temperatures the onset shows broadening and we found an increase in the onset energies of the phonons towards lower side with increasing temperature. The quantitative explanation of this phonon structure was given by Suemoto et al (1993). In this resonant excitation, the PL spectrum results from the superposition of the emission lines of all the quantum particles excited with different efficiencies. A quantitative evaluation of these onset energies suggests that their source has a phonon spectrum and the optical selection rules are similar to that of crystalline silicon. The onsets in our resonantly excited PL spectra are due to the optical transitions involving the emission of 0,1 , and $2 \mathrm{TO}(\Delta)$ momentum-conserving phonons with weak features associated with weakly coupled $\mathrm{TA}(\Delta)$ phonons. Our experimental results describe the two luminescent material model by Rosenbauer et al (1995), where PL originates from quantum-confined crystalline $\mathrm{Si}$ with resonant excitation at $647 \mathrm{~nm}$ and from unspecified additional luminescent material with excitation in the range $514-488 \mathrm{~nm}$. As all the experimental aspects of the phonon structures and PL mechanism cannot be explained consistently with a specified model, more elaborate experimental and theoretical analysis are needed to confirm the luminescence in Si nanostructures and porous silicon. In order to explain the PL mechanism in the $\mathrm{Si}$ nanostructures due to quantum confinement in the near surface region, we calculate the band gap that is sensitive to the surface effects and the shape of the silicon quantum dots. The theoretical calculations are based on the empirical pseudopotential method (EPM) for the electronic structure of silicon quantum dots of different sizes. Following the model of Wang and Zunger (1994), we use the local pseudopotential of the form

$$
V_{\mathrm{Si}}=\frac{a_{1}\left(q^{2}-a_{2}\right)}{a_{3} e^{a_{4} q^{2}}-1},
$$

with $a_{1}=0 \cdot 2685, a_{2}=2 \cdot 19, a_{3}=2 \cdot 06$, and $a_{4}=0.487$ in the atomic units (Hartree for energy, $\mathrm{Bohr}^{-1}$ for momentum, q). The hydrogen empirical pseudopotential (in atomic units) is given by

$$
\begin{aligned}
V_{\mathrm{H}}(q)= & -0 \cdot 1416+0.009802 q+0.06231 q^{2} \\
& -0.01895 q^{3} ; \text { when } q \leq 2, \\
V_{\mathrm{H}}(q)= & +\frac{0.02898}{q}-\frac{0.3877}{q^{2}}+\frac{0.9692}{q^{3}}-\frac{1.022}{q^{4}} ; \\
& \text { when } q>2 .
\end{aligned}
$$

We used an ideal unrelaxed structure with $\mathrm{Si}-\mathrm{H}$ bond distance of $1.487 \AA$ for all the cases. We expand the dot wavefunctions in a large basis of plane waves as

$$
\psi_{\mathrm{j}}(r)=\Sigma_{\mathrm{G}} B_{\mathrm{j}}(\mathbf{G}) e^{i G \cdot r}
$$

where, $\mathbf{G}$ is a reciprocal vector and $B_{\mathrm{j}}(\mathbf{G})$ are the expansion coefficients. To find the size dependence of the gap energy and the near band gap solutions i.e. the separation between the highest occupied molecular orbital (HOMO) and the lowest unoccupied molecular orbital (LUMO), we solve the effective single-particle Schrödinger equation for different sizes of the quantum dot. We use a cut off energy of $5 R_{\mathrm{y}}$ for the plane wave expansion and a silicon bulk lattice constant of $5.43 \AA$ for the smallest dot. We find all eigenvalues by direct diagonalization method. The transformation between $\psi_{\mathrm{j}}(\mathbf{r})$ on a real space grid and $B_{\mathrm{j}}(\mathbf{G})$ on a reciprocal space grid is done by numerical fast

\begin{tabular}{|c|c|c|c|c|}
\hline $\begin{array}{l}\text { Number of } \\
\text { atoms in } \mathrm{Si} \\
\text { cluster }\end{array}$ & Arrangement of $\mathrm{Si}$ atoms & $\begin{array}{l}\text { Fixed bond length } \\
\text { of } \mathrm{Si} \text { atoms } \\
(\AA)\end{array}$ & $\begin{array}{l}\text { Gap energy } \\
\text { without } \mathrm{H} \\
(\mathrm{eV})\end{array}$ & $\begin{array}{l}\text { Gap energy } \\
\text { with } \mathrm{H} \\
(\mathrm{eV})\end{array}$ \\
\hline 5 & Five atoms in tetrahedral arrangements & $5 \cdot 43$ & 1.997 & $2 \cdot 07$ \\
\hline 8 & $\begin{array}{l}\text { Eight atoms joined by single bond with an } \\
\text { appropriate dihedral angle }\end{array}$ & $4 \cdot 00$ & $1 \cdot 820$ & $1 \cdot 89$ \\
\hline 17 & $\begin{array}{l}\text { Seventeen atoms cluster with four tetra- } \\
\text { hedrally arranged nearest neighbours and } \\
\text { twelve neighbours at fcc centres }\end{array}$ & $3 \cdot 20$ & $1 \cdot 790$ & $1 \cdot 85$ \\
\hline 18 & $\begin{array}{l}\text { Eighteen atoms cluster using a cubic cell } \\
\text { with eight corner atoms, six face centred } \\
\text { atoms and four from other sub-lattice entirely } \\
\text { included in it }\end{array}$ & $3 \cdot 00$ & $1 \cdot 690$ & $1 \cdot 82$ \\
\hline
\end{tabular}
Fourier transform (FFT). The numerical results for the four different sizes and geometries of the clusters are given in table 1 . The value of the gap energy decreases as the size of the cluster increases, which implies a weaker

Table 1. Calculated gap energy of Si clusters with different sizes (with and without hydrogen at the surface). 


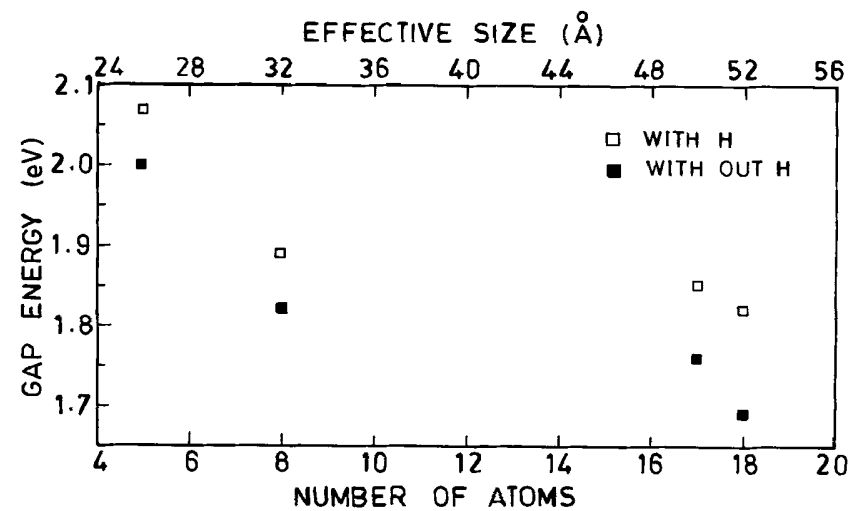

Figure 5. Plots of gap energy as a function of number of $\mathrm{Si}$ atoms and effective size of the cluster. The open and closed squares represent the gap energy of $\mathrm{Si}$ dots with and without hydrogen atoms.

confinement for large sizes. As we saturate the surface with hydrogen atoms, there is an enhancement of gap energy in the range $0 \cdot 08-0 \cdot 13 \mathrm{eV}$ as we go from 5 to 18 atoms cluster. The variation of gap energy for different sizes of the dot with and without hydrogen passivated surfaces is shown in figure 5. Here we have calculated the effective size of the dot under linear mapping $d=N a(\AA)$ only to get a proper scaling of our data points. We find the gap energy increases as the size of the dot decreases, which confirms the stronger confinement for the smaller sizes. The higher value of the gap energy in the presence of hydrogen is due to strong $\mathrm{Si}: \mathrm{H}$ bonds which has a confinement effect in the near surface region.

The electronic states in Si cluster can be classified into three categories: (i) delocalized states that experience full confinement effect, (ii) strongly localized states with spatial distribution smaller than the cluster diameter. Their energy lies deep into the gap and are insensitive to the confinement effect thus exhibit no blue shift, and (iii) weakly localized states with spatial distribution of the order of the cluster diameter with energy near the gap and show intermediate blue shift. The apparent blue shift in amorphous silicon clusters thus has two origins (i) the varying proportion of clusters on strongly localized states and (ii) the usual confinement effect on the other states. Delerue et al (1999) obtained a two-peak density of state distribution corresponding to strongly and weakly localized or delocalized states in larger amorphous Si clusters. In hydrogenated clusters they show only the normal confinement effect and HOMO-LUMO gap values closer to the $c$-Si clusters. Similar results have also been obtained by Allan et al (1997) for the average HOMO-LUMO gap vs size for layers. It must be emphasized that the experimental results do not critically depend on the presence of hydrogen in the layer while theoretical calculations show clear distinction between hydrogen free and hydrogenated clusters.

\section{Conclusions}

The optical properties of Si nanocrystallites and Si clusters prepared by different techniques were investigated using PL and Raman scattering. The PL shows broad luminescence band in the red region from $\mathrm{Si}$-doped $\mathrm{SiO}_{2}$ thin films prepared by co-sputtering of $\mathrm{Si}$ and $\mathrm{SiO}_{2}$, annealed in $\mathrm{Ar}$ and $\mathrm{O}_{2}$ atmosphere. The nanocrystalline silicon particles prepared by pulsed plasma technique shows infrared PL band. The Raman spectra from this sample show a broad band superimposed on a sharp line and is associated with amorphous silicon like structure in the film. Similar investigations in porous silicon were done to correlate the optical properties of Si nanostructures prepared by different techniques. The resonantly excited PL spectra shows step-like phonon structures, which corresponds to different luminescence mechanism in porous silicon. In order to explain the luminescence results, electronic structure of 5-18 atoms Si quantum dots is calculated using empirical pseudopotential method. The calculated gap energy is enhanced when the dot is passivated with hydrogen atoms incorporating proper hydrogen pseudopotential.

\section{Acknowledgements}

We would like to thank Prof. S Nozaki, University of Electrocommunications, Japan and Prof. S Oda, Tokyo Institute of Technology, Japan for providing $\mathrm{Si}$-doped $\mathrm{SiO}_{2}$ thin film and nanocrystalline $\mathrm{Si}$ samples.

\section{References}

Allan G, Delerue C and Lannoo M 1997 Appl. Phys. Lett. 71 1189

Canham L T 1990 Appl. Phys. Lett. 571046

Campbell I H and Fauchet P M 1986 Solid State Commun. 58 739

Delerue C, Allan G and Lannoo M 1999 J. Lumin. 8065

John G C and Singh V A 1995 Phys. Rep. 26393

Kanemitsu Y 1995 Phys. Rep. 2631

Morisaki H, Ping F W, Ono H and Yazawa K $1991 \mathrm{~J}$. Appl. Phys. 701869

Osaka Y, Tsunetomo K, Toyomura F, Myoren H and Kohno K 1992 Jpn J. Appl. Phys. 31 L365

Rosenbauer M, Finkbeiner S, Bastarret E, Weber J and Stutzmann M 1995 Phys. Rev. B51 10539

Suemoto T, Tanaka K, Nakajima A and Itakura T 1993 Phys. Rev. Lett. 703659

Takagahara T and Takeda K 1996 Phys. Rev. B53 R4205

Wang L W and Zunger A 1994 J. Chem. Phys. 1002394 